\title{
Direct-Written Printed Dual-Polarized Meshed Antenna for Chipless RFID Sensing
}

\author{
Mahmoud Wagih and Junjie Shi \\ School of Electronics and Computer Science, University of Southampton, SO17 1BJ, Southampton, U.K. \\ http://ecs.soton.ac.uk/
}

\begin{abstract}
This paper presents a dual-polarized coplanar waveguide (CPW) monopole antenna, fabricated using additive manufacturing on a flexible substrate for chipless RFID tags. The proposed antenna is based on a dual-port broadband disc monopole with a CPW feed and a notched ground plane for mutual coupling suppression, occupying over $50 \%$ less area compared to a dual-antenna tag. The antenna is meshed achieving a $90 \%$ transparency and is fabricated using dispenser printing on a flexible polyimide substrate. Through a ground plane notch and using meshed conductors, the antenna's total efficiency is improved by $30 \%$ over a standard dual-port disc. The antenna maintains a simulated and measured $S_{11}$ bandwidth form 1.4 to $2.55 \mathrm{GHz}$. With over $60 \%$ and up to $75 \%$ radiation efficiency while being $90 \%$ optically-transparent, the antenna is suitable for chipless RFID applications in the sub- $6 \mathrm{GHz}$ UHF spectrum.
\end{abstract}

\section{Introduction}

Chipless RFID sensor tags have attracted significant research interest for future internet of things (IoT) applications $[1,2]$. Owing to their low-cost and and suitability for mass productions, multiple sensors have been proposed for chipless RF-based sensing [2]. For instance, gas [3] and humidity sensors [4] have been developed based on chipless tags directly printed or etched on flexible substrates. Furthermore, emerging applications such as food monitoring directly benefits from chip-less RF sensing [2].

Additive manufacturing is a low-cost method for realizing RF components and antennas [5]. For example, directwrite printing can be utilized to prototype and realize highefficiency RF energy harvesters [6]. In addition, multiple thin-film sensors, such as a carbon-nanotube (CNT) chipless RFID sensor have been proposed using printed electronics [3]. Meshed antennas [7], widely used for opticallytransparent antennas [8], can be used to reduce the costs of printed antennas used in chipless RFID tags, by reducing the amount of conductive ink without detrimentallyaffecting the antenna's performance [6].

In most Ultra-Wide Band (UWB) chipless RFID tag implementations, a pair of antennas are used to receive and

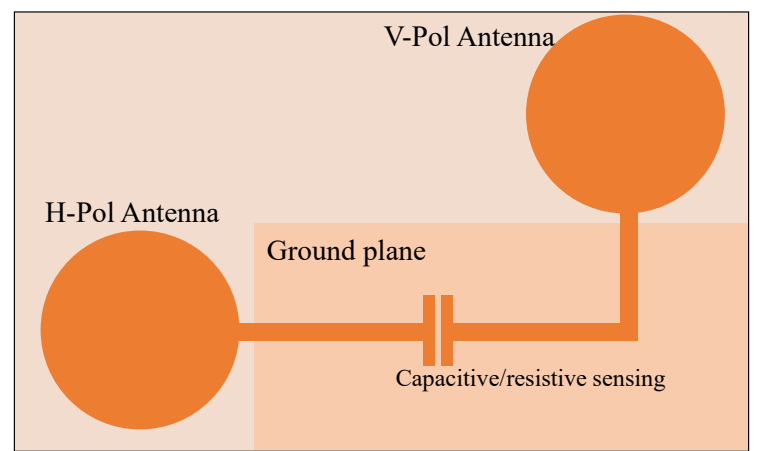

(a)

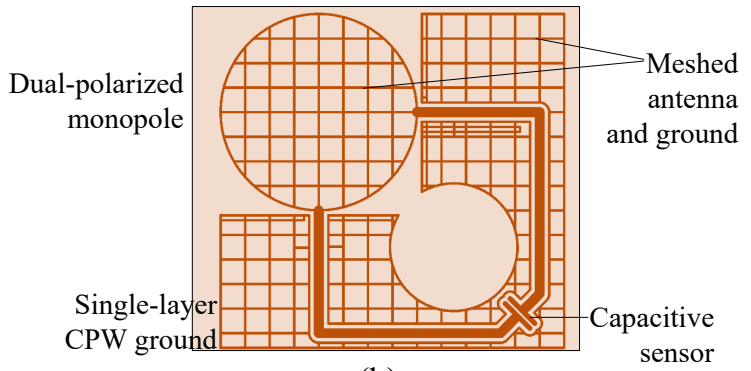

(b)

Figure 1. Layout of a typical chipless RFID with separate antennas (a) and the a chipless RFID sensor tag based on the proposed single-layer meshed dual-polarized antenna (b).

reflect and the incident signal from the reader, with an orthogonal polarization $[1,9,10]$. Disc antennas have been widely used in chipless RFID tags owing to their simple geometry and wide bandwidth [9]. On the other hand, this increases the size of the tag making its integration in Internet of Everything (IoE) systems more obtrusive. Therefore, a need for compact RFID tags with dual-polarized antennas compatible with additive manufacturing.

In this paper, a dual-port dual-polarized coplanar waveguide (CPW) monopole antenna is proposed for compact chipless RFID sensing tags. The proposed antenna is designed to minimize the undesirable mutual coupling between the ports over a standard dual-port antenna and is fabricated using direct-write dispenser printing on a flexible substrate. The antenna and the ground plane are meshed to reduce the fabrication cost and enable optically-transparent tags, showing no noticeable degradation in the $S_{11}$ or the 

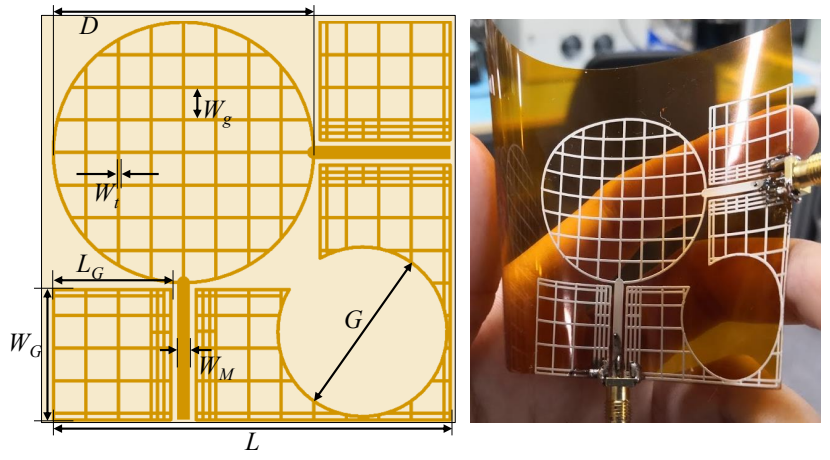

Figure 2. Layout and photograph of the proposed dualpolarized antenna

radiation properties. Section 2 presents the antenna design and simulation. The antenna is fabricated and characterized experimentally in Section 3.

\section{Meshed Dual-Polarized Monopole Design and Simulation}

A typical chipless RFID sensor tag is shown in Figure 1a, composed of two orthogonally-polarized antennas and a microstrip line where the tag's ID or sensing elements are incorporated [9]. The proposed antenna, shown in Figure 1$\mathrm{b}$, is based on a dual-port disc monopole. The antenna is designed with a CPW feed to be directly printed on a single layer. The antenna was designed with a $\lambda / 4$ diameter to maintain a wide bandwidth. By using a single radiating element, the tag's size can be significantly reduced as opposed to a dual-polarized

A key challenge with a broadband monopole is the mutual coupling between the ports, which can significantly reduce the realized gain of the antenna. It was shown that a slotted ground plane can be introduced to reduce the coupling between the antenna's ports [11]. A circular notch is added to the ground plane as shown in Figure 2. The diameter of the antenna was calculated as $D=\lambda_{0} / 4$ at $1.25 \mathrm{GHz}$; the dimensions of the fabricated antenna are mm: $D=40, L=61$, $G=25, W_{G}=20, L_{G}=18, W_{M}=2$.

The antenna was simulated in CST Microwave Studio to investigate its s-parameters. Figure 3 shows the simulated $S_{11}$ of the dual-port antenna with a $S_{11}<-10 \mathrm{~dB}$ bandwidth from 1.25 , closely agreeing with the $1.25 \mathrm{G} \mathrm{MHHz}$ $\lambda / 4$ disc $D$. Nevertheless, the high port coupling $\left(S_{21}\right)$ will result in efficiency degradation. After the notch of diameter $G$ is introduced, the mutual coupling is suppressed by $5 \mathrm{~dB}$ at $1.5 \mathrm{GHz}$, ad over $10 \mathrm{~dB}$ above $2.4 \mathrm{GHz}$.

For a meshed antenna, the theoretical optical transparency of the antenna can be calculated using

$$
\text { Transparency }=\left(1-\frac{W_{t}}{W_{g}+W_{t}}\right)^{2}
$$
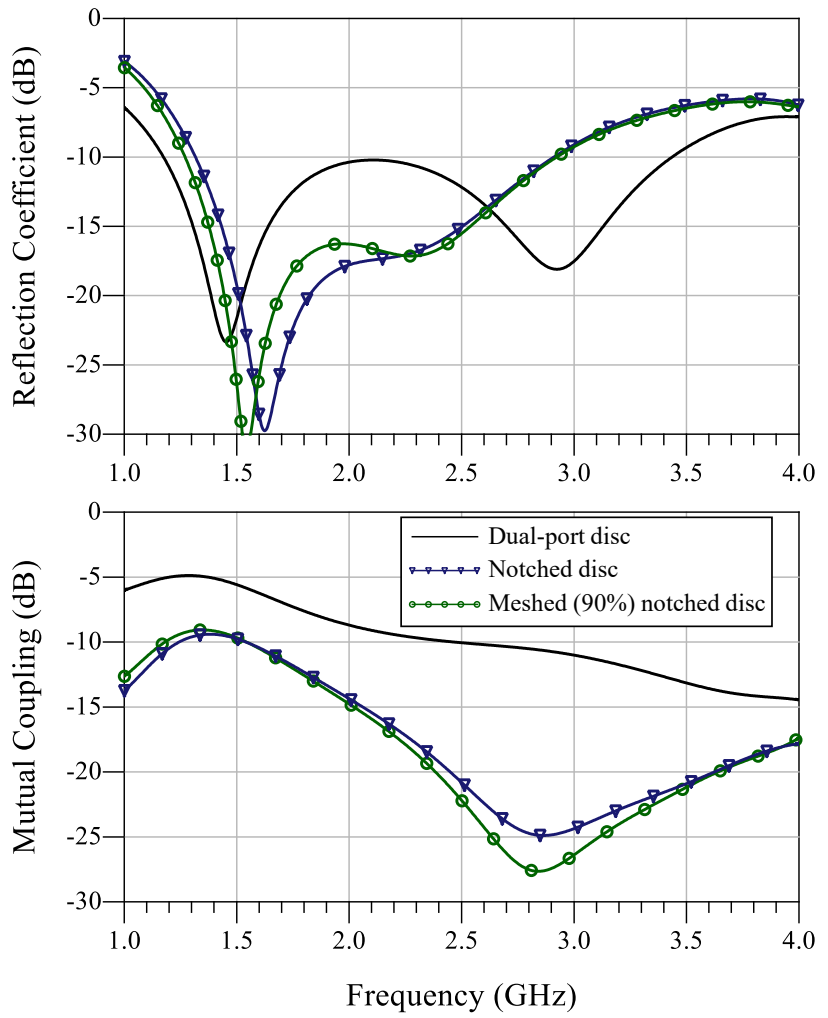

Figure 3. Simulated reflection coefficient and port mutual coupling of the dual-port disc antenna before and after introducing the notch and meshing.

where $W_{t}$ and $W_{g}$ are the trace and gap width, respectively [8]. $W_{g}=5 \mathrm{~mm}$ was selected to achieve around $90 \%$ transparency, where $W_{t}=0.28 \mathrm{~mm}$ and is limited by the fabrication method, discussed in the next section.

In Figure 3, it can be observed that the meshed conductors had a very small impact on the $S_{11}$ response, causing under $20 \mathrm{MHz}$ shift in the antenna's lower bandwidth. This can be explained by the larger mesh gaps introducing additional non-uniformities which may cause radiation at a lower frequency. As for the mutual coupling, the meshed conductors improve the port isolation by approximately $2 \mathrm{~dB}$ around $2.7 \mathrm{GHz}$.

The radiation properties of the antenna have been simulated in CST over the antenna's bandwidth. Figure 4 shows the simulated total efficiency (inclusive of mismatch) of the antenna, showing around $3 \mathrm{~dB}$ relative efficiency improvement after the ground-plane notch is introduced. In addition, it can be observed that despite being meshed to use 90\% less conductive ink, the antenna maintains a stable efficiency before and after meshing, showing that meshed antennas are suitable for broadband applications. The antenna maintains a gain over $1.8 \mathrm{dBi}$ from $1.5 \mathrm{GHz}$, with over $12 \mathrm{~dB}$ co/cross-polarization isolation over both ports, showing its suitability for dual-polarized chipless RFID. Figure 5 shows the simulated radiation patterns over the elevation planes. It can be observed that for both 1.5 and $2.4 \mathrm{GHz}$, 


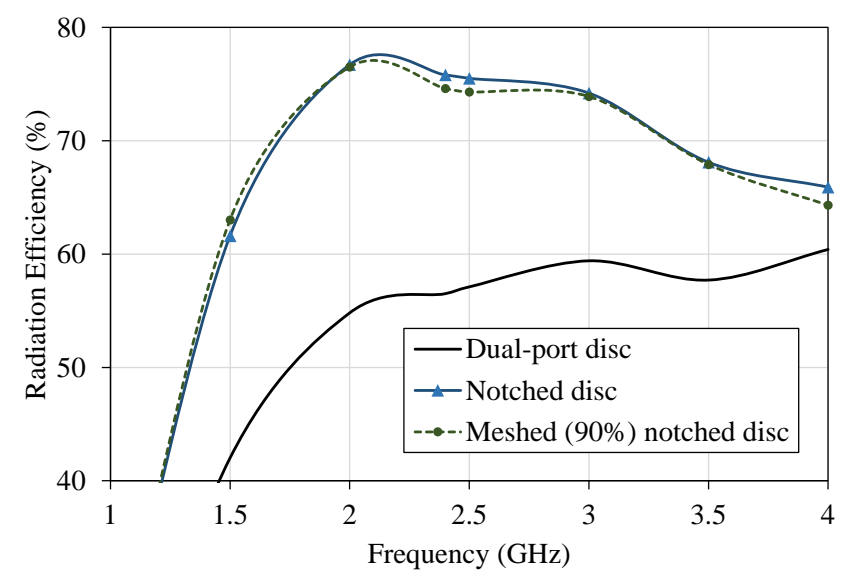

Figure 4. Simulated radiation efficiency of the dualpolarized antenna for different design steps.

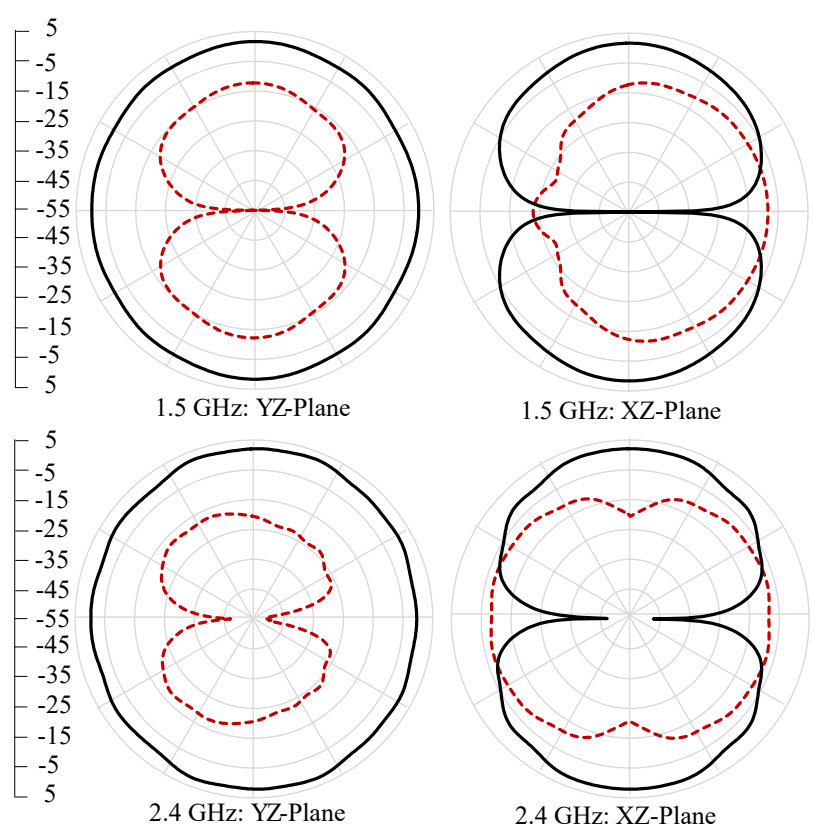

Figure 5. Simulated radiation efficiency of the dualpolarized antenna for different design steps.

the radiation patterns do not vary with frequency, where the co/cross-polarization is maintained over the antenna's main beam.

\section{Antenna Fabrication and Measurements}

The antenna was fabricated using direct-write dispenser printing on a flexible $75 \mu \mathrm{m}$-thick polyimide substrate. A conductive silver ink (Voltera flex conductor) was used for the antenna traces, with a measured resistance around $0.01 \Omega /$ square. The traces were printed using a commercial dispenser printer, Voltera V-One, as in Figure 6-a. Following the ink deposition, a standard hot-plate is used to cure the ink at $170^{\circ} \mathrm{C}$ for 50 minutes. Once the ink is cured, the flexible polyimide film is removed from the planar carrier substrate and the SMA connector is mounted using lowtemperature solder, as in Figure 6-c.

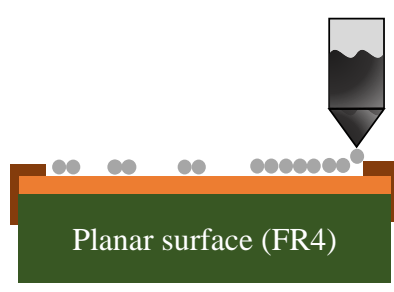

(a)

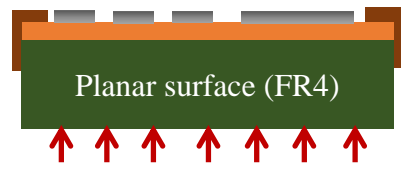

(b)

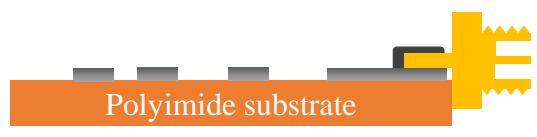

(c)

Figure 6. Simulated (dashed) and measured (solid) sparameters of the $88 \%$-transparent antenna prototype.

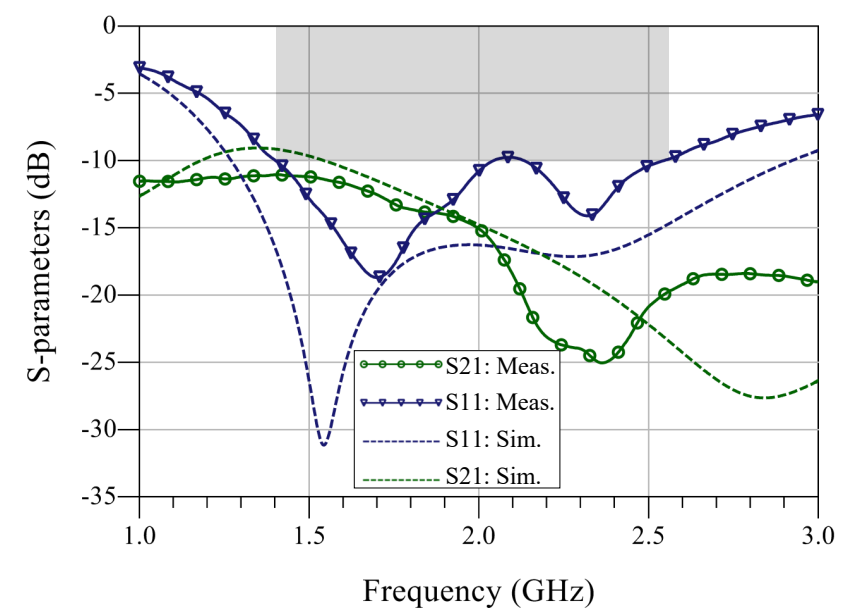

Figure 7. Simulated (dashed) and measured (solid) sparameters of the $88 \%$-transparent antenna prototype.

The fabricated dual-port prototype, shown in Figure 2, was measured using a TOSM-calibrated Rohde and Schwarz ZVB4 vector network analyzer (VNA). Figure 7 shows the simulated and measured two-port s-parameters of the $90 \%$ transparent antenna prototype.

From Figure 7, it can be observed that the antenna maintains an $S_{11}<-10 \mathrm{~dB}$ bandwidth from 1.4 to $2.55 \mathrm{GHz}$, a $58 \%$ fractional bandwidth. This demonstrates tha antenna's suitability for broadband chipless RFID sensing applications. The narrower bandwidth compared to the simulation is due to the variations introduced by the SMA connectors and the non-uniformity of the solder termination.

As for the ports' mutual coupling, it can be observed from the measured $S_{21}$ that the undesired port coupling is under $-10 \mathrm{~dB}$ over the antenna's full bandwidth, and is around $-25 \mathrm{~dB}$ in the $2.4 \mathrm{GHz}$ license-free band. This demonstrates that, should the antenna be used for re-transmitting signals in a sensor tag, the high-isolation between the two ports guarantees that the antenna's realized gain will not degrade. This further validated the total efficiency improve- 
ment observed in simulation, as previously shown in Figure 4.

\section{Conclusion}

In this paper, a compact dual-polarized CPW antenna was presented using additive manufacturing on a thin and flexible organic substrate, for chipless RFID applications. The proposed antenna maintains a $58 \%$ fractional bandwidth from 1.4 to $2.55 \mathrm{GHz}$ and a broadband radiation efficiency over $70 \%$ above $1.7 \mathrm{GHz}$. Furthermore, it was shown that the $90 \%$ theoretical optical transparency achieved through meshing does not have a detrimental impact on the antenna's $S_{11}$ bandwidth or total efficiency, concluding that meshed transparent antennas are suitable for broadband chipless RFID tags. Future investigations include adding coupled resonator elements to the antenna's CPW feed for the tag's ID, in addition to utilizing smart dielectric and conductive materials such as CNTs for passive wireless capacitive and resistive sensing.

\section{Acknowledgements}

This work was supported by the UK Engineering and Physical Sciences Research Council (EPSRC) under Grant EP/P010164/1.

\section{References}

[1] S. Preradovic and N. C. Karmakar, "Chipless RFID: Bar Code of the Future," IEEE Microw. Mag., vol. 11 no. 7 , pp. $87-97,2010$.

[2] E. M. A. J. K. S. N. C. Karmakar, "Smart Sensing Materials for Low-Cost Chipless RFID Sensor," IEEE J. Sensors, vol. 14 no. 7, pp. 2198 - 2207, 2014.

[3] L. Yang, R. Zhang, D. Staiculescu, C. P. Wong, and M. M. Tentzeris, "A Novel Conformal RFID-Enabled Module Utilizing Inkjet-Printed Antennas and Carbon Nanotubes for Gas-Detection Applications," IEEE Antennas Wireless Propag. Lett., vol. 8, pp. 653 -656, 2009.

[4] J. G. D. Hester and M. M. Tentzeris, "Inkjet-Printed Flexible mm-Wave Van-Atta Reflectarrays: A Solution for Ultralong-Range Dense Multitag and Multisensing Chipless RFID Implementations for IoT Smart Skins," IEEE Trans. Microw. Theory Techn., vol. 64,12 , pp. 4763 - 4773, 2016.

[5] S. A. Nauroze, J. G. Hester, B. K. Tehrani, W. Su, J. Bito, R. Bahr, J. Kimionis, and M. M. Tentzeris, "Additively Manufactured RF Components and Modules: Toward Empowering the Birth of Cost-Efficient Dense and Ubiquitous IoT Implementations," Proc. IEEE, vol. 105, no. 4, pp. 702 - 722, 2017.
[6] M. Wagih, A. S. Weddell, and S. Beeby, "Meshed High-Impedance Matching Network-Free Rectenna Optimized for Additive Manufacturing," IEEE Open Journal of Antennas and Propagation, vol. 1, pp. 615 -626, 2020.

[7] G. Clasen and R. Langley, "Meshed patch antennas," IEEE Trans. Antennas Propag., vol. 52 no. 6, pp. 1412 - 1416, 2004.

[8] Z. J. Silva, C. R. Valenta, and G. Durgin, "Optically Transparent Antennas : A Survey of Transparent Microwave Conductor Performance and Applications," IEEE Antennas Propag. Magazine, vol. Early Access, 2020.

[9] S. Preradovic and N. C. Karmakar, "Design of Chipless RFID Tag for Operation on Flexible Laminates," IEEE Antennas Wireless Propag. Lett., vol. 9, pp. 207 $-210,2010$.

[10] R. R. G. E. B. S. Bhadra, "Wireless Passive Sensors for Food Quality Monitoring: Improving the Safety of Food Products," IEEE Antennas Propag. Mag., vol. 62 no. 5, pp. $76-89,2020$.

[11] M. Wagih, A. S. Weddell, and S. Beeby, "Omnidirectional Dual-Polarized Low-Profile Textile Rectenna with over $50 \%$ Efficiency for Sub- $\mu \mathrm{W} / \mathrm{cm}^{2}$ Wearable Power Harvesting," IEEE Trans. Antennas Propag., vol. Early Access, 2020. 